\title{
MAPLE chip: a processing element for a static scheduling centric multiprocessor
}

\author{
Kenta Yasufuku ${ }^{\dagger}$ \\ Riku Ogawa $^{\dagger}$ \\ $\dagger$ Keio University \\ Dept. of Information and Computer Science \\ 3-14-1, Hiyoshi Yokohama, 223-8522 Japan \\ Tel: $+81-45-560-1063$ \\ Fax: $+81-45-560-1064$ \\ e-mail: \{yasufuku, riku, hunga\}@am.ics.keio.ac.jp
}

\author{
Keisuke Iwai $^{\dagger \dagger}$ \\ Hideharu Amano $^{\dagger}$ \\ ${ }^{\dagger}$ National Defense Academy \\ Dept. of computer science \\ 1-10-20, Hashirimizu Yokosuka, 239-8686 Japan \\ Tel: +81-468-41-3810 \\ Fax: +81-468-44-5911 \\ e-mail: iwai@nda.ac.jp
}

\begin{abstract}
A custom processor called MAPLE which supports static scheduling by automatic parallelizing compilers is implemented and evaluated. MAPLE has a high performance floating point arithmetic unit and fast data transfer mechanism between other MAPLE chip. The maximum operational frequency of MAPLE chip is $80 \mathrm{MHz}$ in simulation, and the operation on the prototype board with $23 \mathrm{MHz}$ clock is confirmed. Its required power consumption is about $0.56 \mathrm{~W}$ at $23 \mathrm{MHz}$ operation.
\end{abstract}

\section{INTRODUCTION}

Although it is easy to enhance the peak performance of the multiprocessor only by adding many processing units, it is difficult to exploit effective performance for users without support of automatic parallelizing compilers. However, such compilers have been tailored for existing multiprocessors which are designed without care of them well. The multiprocessor system ASCA(Advanced Scheduling oriented Computer Architecture)[1] has been proposed based on the idea that not the parallelizing software is tailored for machines, but a multiprocessor system should be designed to make the best use of parallelizing software.

In ASCA, a multi-grain parallelizing compilation scheme[2] is adopted. The scheme can exploit parallelism of the user program in various levels of granularity: coarse-grain parallelis$\mathrm{m}$ (macro-data flow computation), medium-grain parallelism (loop-level parallelism) which is used in most of the current compilers, and near-fine-grain parallelism (statement level parallelism). Since the near-fine-grain parallelizing compilation especially requires a precise static scheduling between operations, uncertain behavior of the processor must be completely excluded. To solve the problem, we have proposed the custom processor MAPLE[1](Multiprocessor system ASCA Processing eLEment).

\section{CHIP FEATURES}

MAPLE is a 32-bit RISC processor which provides a simple structure with highly predictable operations. Its instruction set is an extension of that of DLX[3]. Five-stage pipeline structure of MAPLE is designed to execute every operation in a fixed number of clocks. A floating-point execution unit[4] includes 32-bit/64-bit IEEE std 754-1985 floating-point unit.
There are 32 integer registers and 32 floating point registers. Furthermore 16 32-bit special registers called receive registers for fast data transfer between other MAPLE chip are provided.

The receive register is directly connected to MAPLE pipeline ID stage, and when the source processor executes a transfer operation between registers, data is directly sent out from the MEM stage of the pipeline. The transferred data is also directly received by a receive register of destination processor.

Fig. 1 illustrates the data transfer between other MAPLE chip by using receive registers.

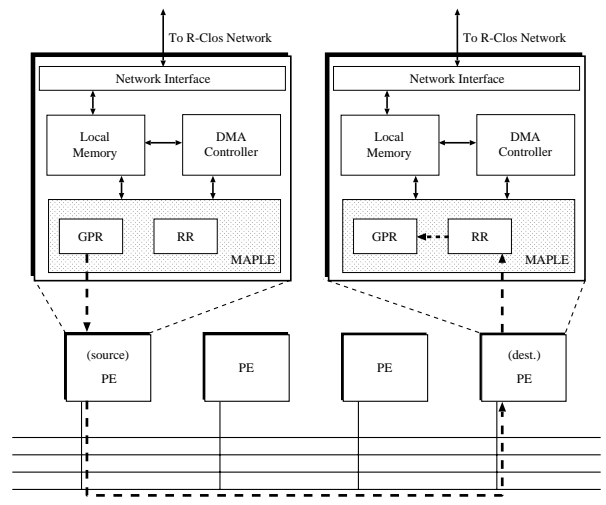

Fig. 1. Data Transfer with Receive Register(RR)

\section{IMPLEMENTATION}

The MAPLE chip in this study has been fabricated in the chip fabrication program of VLSI Design and Education Center(VDEC), the University of Tokyo with the collaboration by Rohm Corporation and Toppan Printing Corporation.

The chip specification and required gates are shown in Table I and Table II. The static delay path analysis results show that the operational frequency of this MAPLE chip is 80MHz. Fig. 2 shows its layout and the packaged die.

TABLE I

THE SPECIFICATION OF MAPLE

\begin{tabular}{|c|c|c|}
\hline Technology & Die size & Package \\
\hline $\begin{array}{c}\text { Rohm CMOS 0.35 } \mu \mathrm{m} \text { Std.Cell } \\
\text { Poly 2, Metal 3, Vdd=3.3V }\end{array}$ & $14.2 \mathrm{~mm} \times 14.2 \mathrm{~mm}$ & PGA 572pin \\
\hline
\end{tabular}


TABLE II

NUMBER OF GATES

\begin{tabular}{|l|r|}
\hline Module & Number of Gates \\
\hline Integer Unit & 21,486 \\
\hline Floating Unit & 145,745 \\
\hline Receive Register & 5,254 \\
\hline Others & 1,525 \\
\hline Total & 174,010 \\
\hline
\end{tabular}
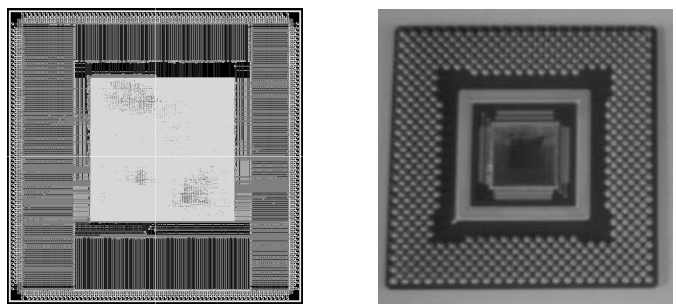

Fig. 2. The MAPLE Cell Layout and packaged Die

\section{EVALUATION}

\section{A. Prototype PE board}

A prototype PE (Processing Element) board with the MAPLE chip is developed as a target of near-fine grain scheduling (Fig. 3). It provides software cache control system, 512kbyte main memory, 32kbyte instruction RAM, 32kbyte flash ROM, and a serial interface. When the system is starting up, a monitor program in the flash ROM runs. Under the management of the monitor program, the user program code is loaded from host computer through the serial interface, and executed.

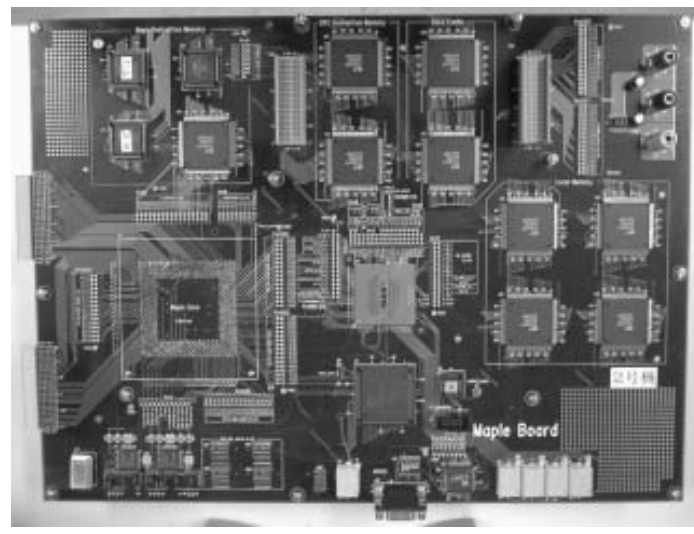

Fig. 3. Prototype PE board

This board is operational at $23 \mathrm{MHz}$ clock which is much less than $80 \mathrm{MHz}$ which is the target frequency of the MAPLE chip. The main reason of the frequency degradation is that the $\mathrm{I} / \mathrm{O}$ pins assignment error was found after the board fabrication, and the MAPLE chip is mounted on a large daughter board for replacing the pin connections, which introduces various electronic problems.

\section{B. Performance}

The performance of the MAPLE chip with $23 \mathrm{MHz}$ clock is evaluated by $\pi$-series-calculation which includes 30,000 iterations, and shown in Table III. For the comparison, the execution result with UltraSPARC-II, which is a chip with similar level of technology, is also shown in the table. While the application runs, the MAPLE chip requires $0.56 \mathrm{~W}$ power consumption.

TABLE III

EVALUATION OF $\pi$-SERIES-CALCULATION

\begin{tabular}{|l|r|r|r|}
\hline CPU & Clock rate[MHz] & Time[ms] & Cycles \\
\hline Sun UltraSPARC-II $^{a}$ & 296 & 48.6 & $14,385,600$ \\
\hline MAPLE $^{a}$ & 23 & 750.0 & $17,250,264$ \\
\hline
\end{tabular}

${ }^{a}$ OS:SunOS 5.8, Compiler:gcc 2.95.3, Compile Option:-O3

${ }^{b}$ Compiler:gcc 2.7.2.3, Compile Option:-O3

The MAPLE chip is designed as a PE for multiprocessor, and we found out that the performance of 4 PEs as 1 cluster is about 2.25 times higher than that of single PE[5]. So, if the MAPLE chip works $80 \mathrm{MHz}$ clock as designed, and runs with 4 PEs, the execution time becomes $95.8 \mathrm{~ms}, 7.83$ times better than the result in the table, and comparable to UltraSPARC-II.

\section{CONCLUSIONS}

The MAPLE chip is an element processor for a static scheduling centric multiprocessor multiprocessor ASCA. Although the performance is lower than UltraSPARC-II, the number of gates and power consumption of the MAPLE chip is so small that the cost/performance and power/preformance of the MAPLE cluster has possibility to contend with the recent supersclar processors.

\section{ACKNOWLEDGEMENTS}

This study is supported by STARC (Semiconductor Technology Academic Research Center, Japan) as the program "A multiprocessor system for the perfect static scheduling."

\section{REFERENCES}

[1] T.Fujiwara, K.Sakamoto, T.Kawaguchi, K.Iwai, H.Amano, "A Custom Processor for the Multiprocessor System ASCA," Applied Informatics '98, 1998, pp258-261.

[2] H.Kasahara, H.Honda, A.Mogi, A.Ogura, K.Fujiwara, S.Narita, "A Multi-Grain Parallelizing Compilation Scheme for OSCAR," 4th Workshop on Languages and Compilers for Parallel Computing, 1991.

[3] J.L.Hennessy and D.A. Patterson, COMPUTER ARCHITECTURE A QUANTITATIVE APPROACH SECOND EDITION, Morgan Kaufmann Publishers, 1996.

[4] T.Kawaguchi, T.Fujiwara, K.Sakamoto, K.Iwai, H.Amano, "Floating Point Arithmetic Unit for the Custom Processor MAPLE," Applied Informatics '99, 1999, pp578-580.

[5] T.Abe, T.Morimura, T.Suzuki, K.Tanaka, M.Koibuchi, K.Iwai, H.Amano, "ASCA chip set: Key components of multiprocessor architecture for multi-grain parallel processing," Proc. of COOL Chips IV, 2001, pp223247. 